Research Article

\title{
Diagnostic Value of CT Angiography Combined with High-Resolution Magnetic Resonance Angiography in Vascular Lesions in Acute Stroke
}

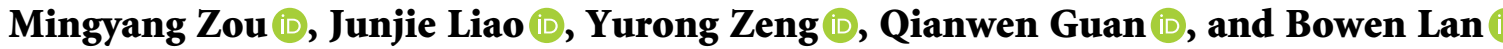 \\ Department of Radiology, Huizhou Municipal Central Hospital, Huizhou 157011, Guangdong, China \\ Correspondence should be addressed to Bowen Lan; 20070572@huanghuai.edu.cn
}

Received 23 June 2021; Accepted 18 August 2021; Published 11 September 2021

Academic Editor: Gustavo Ramirez

Copyright ( $\odot 2021$ Mingyang Zou et al. This is an open access article distributed under the Creative Commons Attribution License, which permits unrestricted use, distribution, and reproduction in any medium, provided the original work is properly cited.

\begin{abstract}
Cerebrovascular disease is increasing rapidly because of its high morbidity and high mortality, which is a serious threat to human health. For the early diagnosis and treatment of diseases, the CT vascular noise combined with high-resolution magnetic resonance angiography in acute cerebral apoplexy vascular disease is adopted. 150 patients with ischemic stroke were selected, which were admitted to the Department of Radiology, Huizhou Central People's Hospital, from January 2020 to December 2020. All patients accepted digital subtraction angiography (DSA), magnetic resonance angiography (MRA), and CT angiography (CTA) examination. Results. There were 76 cases of aneurysm in DSA examination, accounting for $46 \%$; 69 cases with pulsating stenosis, accounting for $50.67 \%$; and 5 cases of moyamoya disease, accounting for $3.33 \%$. The number and proportion of cases of the above diseases in MRA examination were $(75,69,71 ; 53.33 \%, 45.67 \%, 4 \%)$, and those in CTA examination were $(71,76,3$, $47.33 \%, 50.67 \%, 2 \%)$. Relative to the DSA gold standard, the sensitivity, specificity, and false positive rate of MRA were $81.51 \%$, $95.19 \%$, and 2.1, respectively, and those of CTA were $95.78 \%, 79.17 \%$, and 11.0 , respectively. The number of cases and accuracy of detection of cerebral aneurysms by MRA were $(75,96.57 \%)$, and those by CTA were $(71,91.2 \%)$, which was not statistically considerable, $P>0.05$. For the number of cases and the detection accuracy of cerebrovascular malformations, MRA was (38, $92.68 \%)$ and CTA was $(37,90.24 \%)$, which was not statistically considerable, $P>0.05$. Conclusion. The detection sensitivity and accuracy of MRA were better than those of CTA, while specific CTA was superior to MRA. The differences between the two detections were substantial $(P<0.05)$, while the sensitivity and false positive rate were not remarkably different $(P>0.05)$. Therefore, the combination of the two detections was of great significance to the diagnosis and treatment of stroke and other vascular diseases.
\end{abstract}

\section{Introduction}

Ischemic stroke is a common disease in the clinical work of neurology and an important cause of disability and death in the elderly. Therefore, research and exploration of an early diagnosis of ischemic stroke is of great significance. At present, it is clinically believed that ischemic stroke within 6 hours is an acute phase. Taking preventive measures in time during this period can greatly benefit patients' postonset prevention and can reduce disability and mortality [1]. Magnetic resonance angiography (MRA) and CT angiography (CTA) have been utilized clinically in the past three years. According to the current situation, the two methods can accurately reflect the blood perfusion in the tissue, and the latter can directly display the main cerebral artery and the strut. This work retrospectively analyzed the diagnostic significance of these two detection methods in the preischemic stroke, compared their advantages and disadvantages, and discussed the significance of the combination of the two methods in the diagnosis and treatment of cerebrovascular diseases.

In recent years, the diagnosis of cerebrovascular disease has made great progress. Digital subtraction angiography (DSA) is considered the "gold standard" for diagnosing cerebrovascular diseases. However, its shortcomings include causing invasive injury, exposure to radiation, and high cost, 
making it impossible for routine inspections. Unlike DSA, MRA and spiral CTA are both noninvasive vascular imaging detection techniques, which can reflect the shape and state of the vessel lumen realistically and appreciably. Therefore, they help patients avoid DSA [2] pain to a certain extent. However, they are not perfect. MRA may have background suppression, and CTA requires injection of contrast agents. There is no consensus on whether the first choice for cerebrovascular diseases is CTA or MRA.

Noninvasive methods to observe the vascular structure of the central nervous system have always been the focus of imaging techniques. Since the mid-1990s, MRA and CTA have challenged traditional angiography techniques [3]. Angiography is the "gold standard" in many aspects of diagnosing vascular diseases, which has irreplaceable advantages in accurately judging the nature and extent of lesions and the relationship with surrounding structures and is not limited to the diagnosis of cerebral and spinal vascular diseases. In recent years, three-dimensional MRA technology and CTA technology have been applied to the examination and diagnosis of cerebral blood vessels, which can intuitively, objectively, and clearly reflect the vascular structure of the central nervous system. Some scholars believed that MRA and CTA examination techniques had more advantages than DSA and may replace it. MRA and CTA play an important role in the diagnosis and preoperative evaluation of cerebrovascular diseases in primary hospitals [4]. In this work, the results of MRA or CTA of 150 patients with cerebrovascular disease were analyzed, so as to explore and study their adoption significance.

\section{Materials and Methods}

2.1. Basic Information of Research Subjects. From January 2020 to December 2020, 150 patients with cerebrovascular disease admitted to the Department of Radiology, Huizhou Central People's Hospital, were selected. The clinical manifestations were as follows: disharmony of sensorimotor, dizziness with mild aphasia, and blurred vision. Among them, 79 were males and 71 were females. They were $45-75$ years old, with an average of $(64 \pm 1.3)$ years old. There were 65 cases of cerebral artery stenosis, 14 cases of moyamoya disease, and 71 cases of cerebral aneurysm.

2.2. Inclusion Criteria. Inclusion criteria are as follows: I: patients were 45 to 75 years old; II: the clinical manifestations, hospital examinations, and imaging examinations were all in line with the criteria for the diagnosis of ischemic acute cerebral infarction; III: after a period of follow-up, the patient was rediagnosed; IV: the interval from onset to hospital treatment was less than $6 \mathrm{~h}$; V: patients with complete clinical data; VI: all patients and their family members agreed to this study and signed informed consent. The project included in this study was approved by the ethics committee of the hospital.

2.3. Exclusion Criteria. Exclusion criteria are as follows: I: patients with abnormal coagulation function; II: patients with severe renal function or metabolic deficiency; III: patients with intracranial tumor or congenital brain malformation; IV: patients with demyelinating disease; V: patients with serious adverse reaction to iodine contrast agent of the testing instrument; VI: the information was not complete; patients could not cooperate with the research or did not sign the consent.

2.4. MRA Examination. The patient lies on the examination bed with the head in front of the head coil. The long axis of the human body was consistent with the long axis of the bed surface, and hands were placed on both sides of the body or chest. The central sagittal plane of the skull should be consistent with the longitudinal axis of the coil as far as possible and perpendicular to the bed surface. The patient lied on the examination bed with his head forward and his head in the coil area of the head. The long axis of the human body and the long axis of the bed surface were kept at the same horizontal plane, and his hands were placed on both sides of the body or crossed flat on the chest. The central sagittal plane of the skull should be kept in the same trade name as the longitudinal axis of the coil as far as possible and placed vertically on the bed surface. The eyes moved in the center of the horizontal axis of the coil and followed the position of the bed surface. The vertical and horizontal cross positioning lights of the cross were aligned with the coil at the midpoint of the vertical and horizontal axis. The center point of the coil was regarded as the center position, which was gathered and locked, and the magnetic field was sent to the center. Scanning method. Step 1: position imaging: the coronal, sagittal, and axial positioning images were collected simultaneously using a rapid imaging sequence. The baseline, method of scanning, and scope of scanning, etc., were determined on the positioning film. Step 2: imaging scope: centered around the Willis ring or lesion. A presaturation band was set at the proximal or distal end of the scanned area to obtain images of veins and arteries, respectively. If angiography was performed, a pressure band should be placed distally. Step 3: imaging examination method: 3DTOFMRI (time-fly method), referred to as time-fly technique. Step 4: adjustment of parameters: imaging field (FOV): $25-30 \mathrm{~cm}$; imaging layer thickness: $2-3 \mathrm{~mm}$; imaging spacing: no spacing; matrix: $265 \times 265,515 \times 515$, etc. Step 5: after image imaging processing, reconstruction method adopted MIP method or surface method, and multiangle reconstruction was implemented.

2.5. CTA Examination. Toshiba Aquilion 64-slice spiral CT scanner was employed. Scanning was performed from skull base to skull tip, with DFOV $20 \mathrm{~cm}$, tube voltage $120 \mathrm{kV}$, tube current $370 \mathrm{~mA}$, substrate $515 \times 515$, layer thickness $0.630 \mathrm{~mm}$, layer spacing $0.630 \mathrm{~mm}$, tube rotation $0.8 \mathrm{~s}$, and actual scanning time 15-18s. Contrast agent Onipiac or Uveen was $250 \mathrm{mg} / \mathrm{mL}$, and the total dosage was about $150 \mathrm{~mL}$. The tumor was injected through the cubital vein with a high-pressure syringe, and the injection speed was $3.0-3.5 \mathrm{~mL}$. Sweep delay was 20-24 s. Maximum intensity projection (MIP) and volume rendering (VR) were used for postprocessing. 


\section{Results}

3.1. Comparison of DSA, MRA, and CTA Examination Results for Vascular Diseases. Figure 1 shows that, in the DSA examination, among 150 cases of cerebrovascular disease, 76 cases had aneurysms, accounting for $46 \%$; 69 cases had pulsatile stenosis, accounting for $50.67 \%$; and 5 cases had moyamoya disease, accounting for $3.33 \%$. Figure 2 shows that, in the MRA examination, there were 75 cases of aneurysm, accounting for 53.33\%; 69 cases of pulsatile stenosis, accounting for $45.67 \%$; and 6 cases of moyamoya disease, accounting for $4 \%$. Figure 3 shows that, in CTA examination, 71 cases had aneurysms, accounting for 47.33\%; 76 cases had pulsatile stenosis, accounting for $50.67 \%$; and 3 cases had moyamoya disease, accounting for $2 \%$.

3.2. Evaluation Results of the Value of Two Diagnostic Methods. In the diagnosis of cerebral artery stenosis, DSA was set as the gold standard. The sensitivity of MRA examination was $81.51 \%$, the specificity was $95.19 \%$, and the false positive rate was $2.1 \%$. The sensitivity of CTA was $95.78 \%$, the specificity was $79.17 \%$, and the false positive rate was $11.0 \%$. In terms of sensitivity, CTA was greatly better than MRA, and the specificity of MRA was superior to CTA. The difference in specificity, sensitivity, and false positive rate between the two detection methods was remarkable $(P<0.05)$. In the diagnosis of cerebral aneurysm, the sensitivity of MRA examination was $95.89 \%$, the specificity was $87.67 \%$, and the false positive rate was $5.2 \%$. The sensitivity of CTA was $86.72 \%$, the specificity was $86.39 \%$, and the false positive rate was $7.4 \%$. Obviously, MRA was remarkably superior to CTA in terms of sensitivity. In addition, the differences in specificity of the two detection methods were considerable $(P<0.05)$, while the differences in sensitivity and false positive rate were not $(P>0.05)$, as presented in Figures 4 and 5.

3.3. Detection Outcome of Cerebral Aneurysms by MRA and CIA. DSA confirmed that there were 76 cases of cerebral aneurysms. Distribution sites: 25 cases of middle cerebral artery, 17 cases of posterior cerebral artery, 10 cases of posterior communicating artery, 12 cases of anterior communicating artery, 8 cases of posterior inferior cerebellar artery, and 4 cases of internal carotid artery. MRA accurately detected 75 cases, the accuracy rate was $96.57 \%$, $P>0.05$, and the difference was not remarkable relative to that of DSA. There were 24 cases of middle cerebral artery, 17 cases of posterior cerebral artery, 10 cases of posterior communicating artery, 11 cases of anterior communicating artery, 9 cases of posterior inferior cerebellar artery, and 4 cases of internal carotid artery. 71 cases were accurately detected by CTA, the accuracy rate was $91.2 \%$, $P>0.05$, and the difference was not substantial in contrast to that of DSA. 22 cases were middle cerebral artery, 16 cases were posterior cerebral artery, 10 cases were posterior communicating artery, 11 cases were anterior communicating artery, 8 cases were posterior inferior

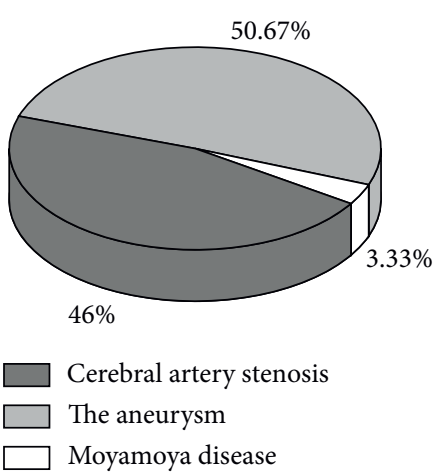

Figure 1: Proportion of various types of diseases in DSA examination results.

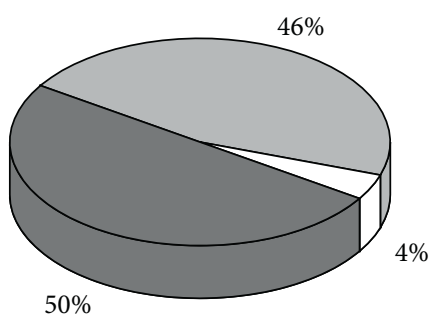

Cerebral artery stenosis

The aneurysm

Moyamoya disease

Figure 2: Proportion of various types of diseases in MRA examination results.

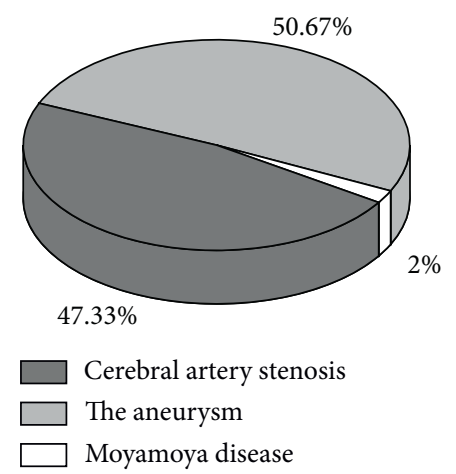

FIgURE 3: Proportion of various types of diseases in CTA examination results.

cerebellar artery, and 4 cases were internal carotid artery. The comparisons are presented in Figures 6 and 7.

3.4. Detection Outcome of Cerebrovascular Malformations by MRA and CIA. As confirmed by DSA, there were 41 cases of cerebrovascular malformations. The distribution locations: 15 cases in frontal lobe, 10 cases in occipital lobe, 9 cases in parietal lobe, 3 cases in temporal lobe, 3 cases in schismatic area, and 1 case in brainstem. MRA accurately detected 38 cases, and the accuracy rate was $92.68 \%$, which was not greatly different relative to that of DSA $(P>0.05)$. The distribution sites: 14 cases of frontal lobe, 10 cases of 


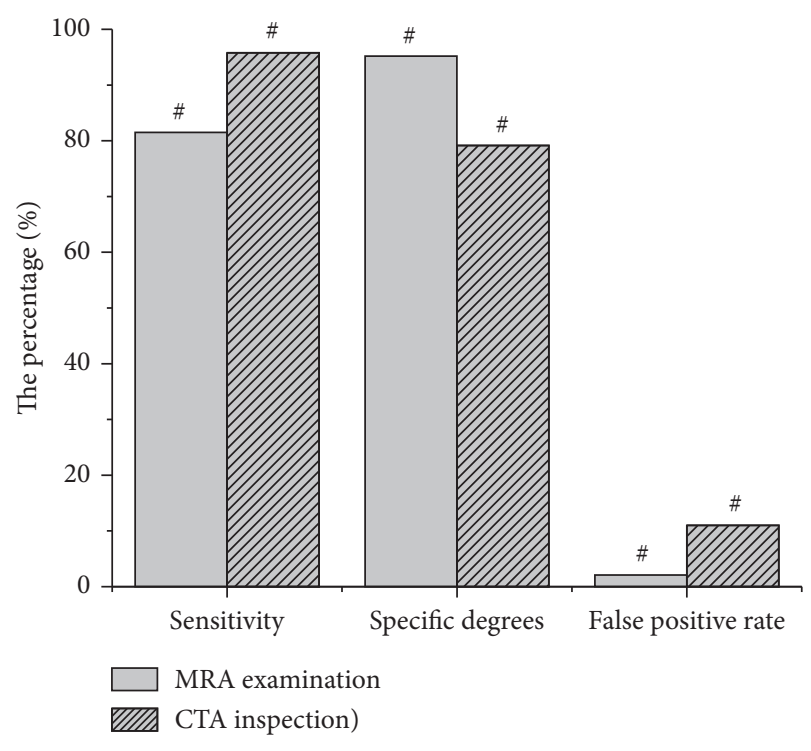

FIgURE 4: Comparison of specificity, sensitivity, and false positive rate of the two methods in the diagnosis of cerebral artery stenosis ( ${ }^{\#} P<0.05$, the difference was statistically considerable).

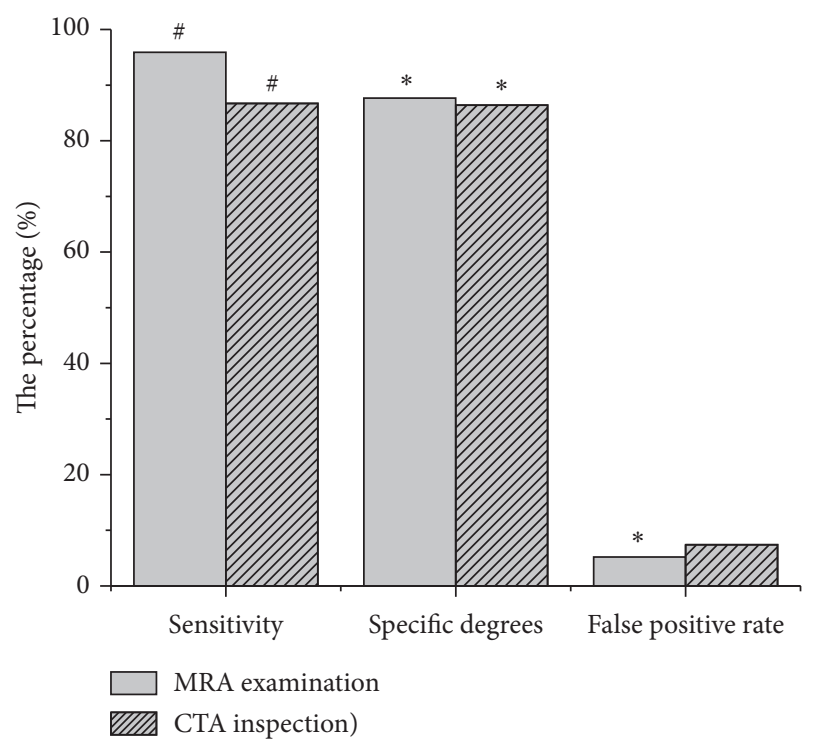

FIgURe 5: Comparison of specificity, sensitivity, and false positive rate of two methods in the diagnosis of cerebral aneurysm $\left({ }^{\#} P<0.05\right.$, the difference was statistically considerable; ${ }^{*} P>0.05$, the difference was not considerable).

occipital lobe, 8 cases of parietal lobe, 3 cases of temporal lobe, 2 cases of scoliosis area, and 1 case of brain stem. CTA accurately detected 37 cases, and the accuracy rate was $90.24 \%$, which was not greatly different relative to that of DSA $(P>0.05)$. The distribution locations: 14 cases of frontal lobe, 9 cases of occipital lobe, 8 cases of parietal lobe, 3 cases of temporal lobe, and 2 cases of scoliosis, and 1 case of brain stem. The details are shown in Figures 8 and 9.

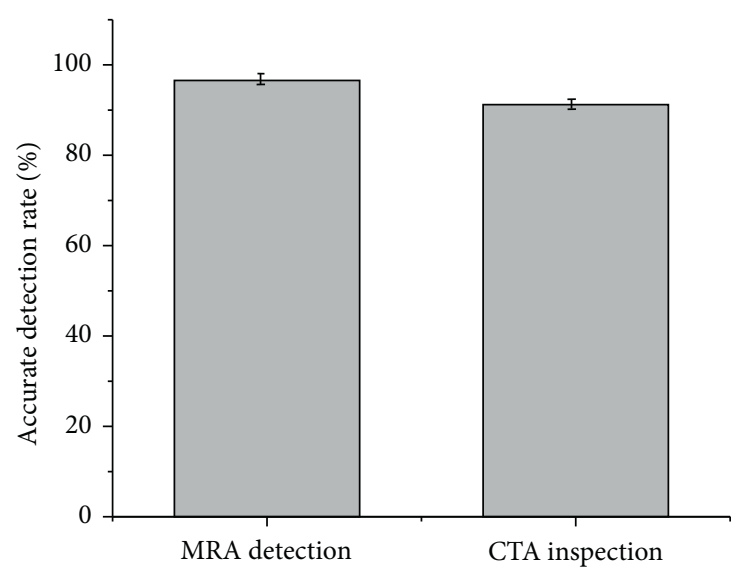

Figure 6: Detection accuracy of MRA and CTA relative to DSA detection $\left({ }^{*}\right.$ in contrast with DSA detection, $\left.P>0.05\right)$.

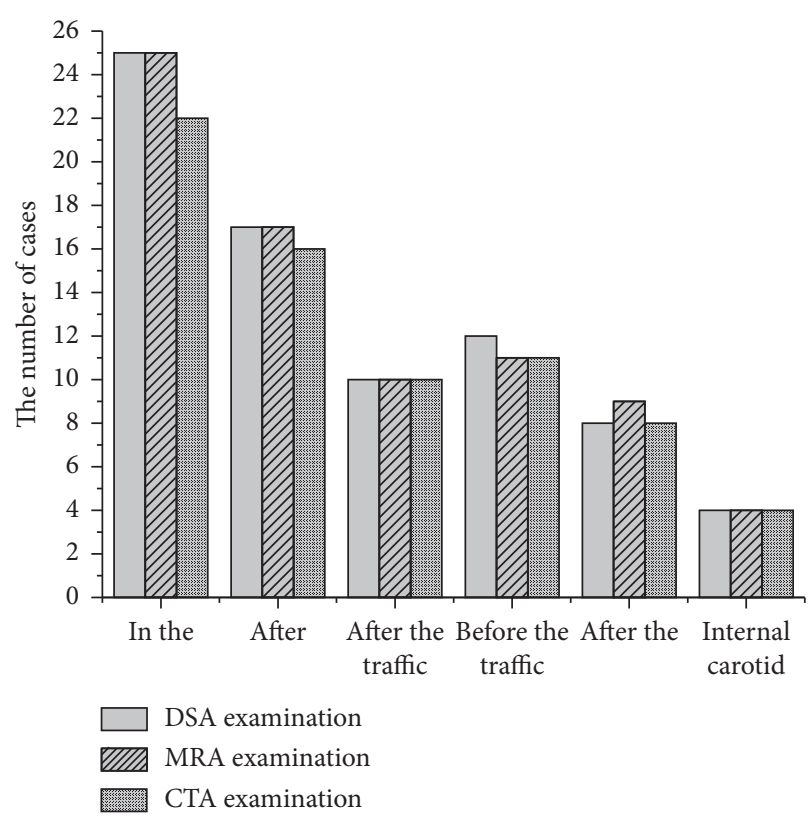

FIGURE 7: The distribution of cerebral aneurysm by each detection method.

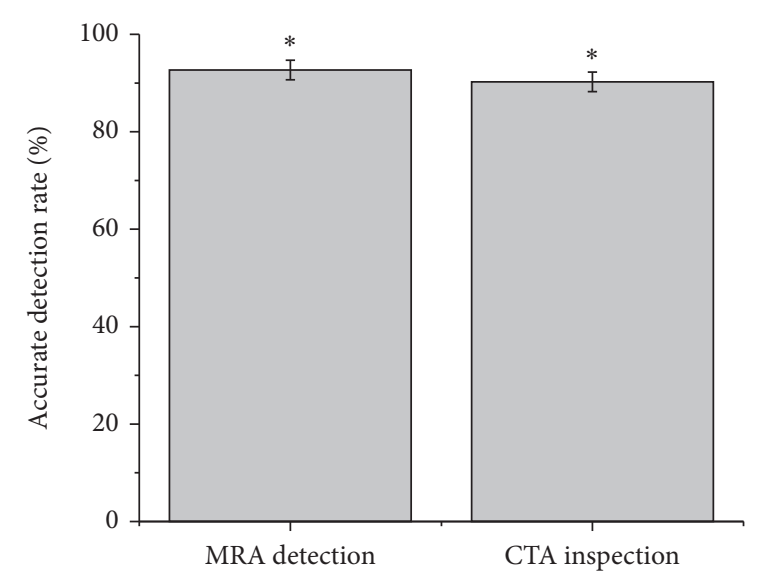

FIgURE 8: Detection accuracy of MRA and CTA relative to DSA detection. 


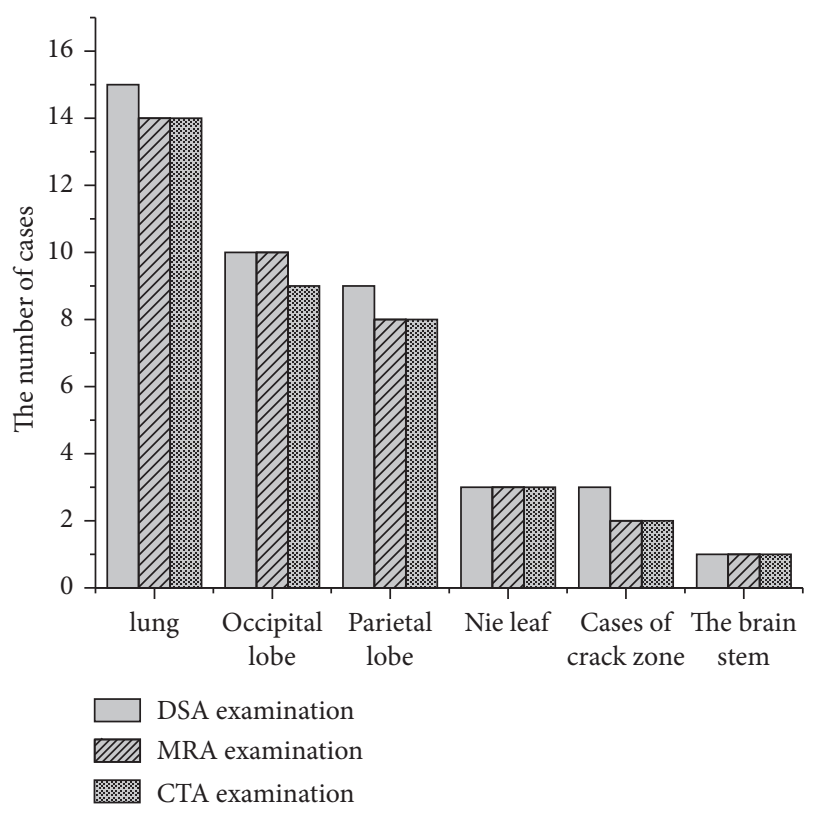

FIgURE 9: The distribution of the detected cerebral vascular malformations by each detection method.

\section{Discussion}

In recent years, the incidence of cerebrovascular disease has been increasing year by year, which has attracted people's attention. Data showed that since 2017, the disease has become the second leading cause of death in humans. With the rapid development of medical science and technology, more and more advanced diagnostic methods have made the diagnosis of cerebrovascular diseases more accurate. However, although there are many diagnostic methods, the clinical efficiency, safety risks, and economic costs of the diagnostic methods should also be considered [5]. Although DSA is highly effective in diagnosing cerebrovascular diseases, its shortcomings are also obvious, and it is generally not used as a very accurate basis for clinical diagnosis of cerebrovascular diseases. Comparing the flow image of blood flow with the static image of surrounding tissues in the natural state, this technique uses the imaging principle of magnetic resonance, which is called MRA. In summary, its basic imaging principle is based on these two effects (the enhancement effect of the mobile phase and the relative position change effect). Then, the relative position comparison method, three-dimensional TOF-MRA technology method, time acceleration method, etc. appear. In general, cerebral arteries were relatively slender and uneven, and TOF-MRA technology was generally appropriate $[6,7]$. Finally, the image after the brain scan is analyzed by computer software, so that the intracranial blood vessels are developed into clear images. CTA is a thin-slice scan of the head after intravenous injection of contrast agent. The image data is input to the computer, and the three-dimensional image reconstruction is performed via a special software package such as volume reconstruction, multiangle plane reconstruction, maximum density projection method, shadow occlusion method, and simulated endoscopy technology, so as to more clearly reflect the cerebrovascular structure. MRA imaging examination and CTA have been widely adopted in clinical practice because they are noninvasive, fast, and simple. However, the spatial resolution of MRA and the accuracy of blood vessel display are low. CTA has some defects in determining the direction of blood flow, it is difficult to completely distinguish the arterial phase and the venous phase, and it is difficult to eliminate the interference of cerebral venous vascular imaging. Therefore, the sensitivity of MRA was lower than CTA in detecting cerebral artery stenosis, but the specificity was higher than CTA [8]. The results were also in line with this research. MRA and CTA examinations have the advantages of no trauma to the human body, fast and efficient, simple, and easy to operate. Therefore, it is very common with clinicians. The spatial resolution and blood vessel display accuracy of MRA are low. However, CTA cannot clarify the direction of blood flow at all, it is difficult to completely distinguish the arteriovenous phase, and it is difficult to exclude the interference of cerebral venography. Therefore, the sensitivity of MRA is lower than that of CTA, and its specificity is higher than that of CTA in diagnosing cerebral artery stenosis. The findings were also consistent with this research. Since TOFMRA technology is not disturbed by venous blood flow, it can clear the abnormal neovascular network of moyamoya disease skull base, and its sensitivity is higher than CTA. MRA is more sensitive to the diagnosis of aneurysms than CTA. The reasons for this phenomenon were analyzed and discussed. MRA can not only be reestablished and rotated at any angle through various methods but also display the position, shape, and size, as well as the relationship between the aneurysm and the carrier artery. It adopted MIP highdensity projection MPR reconstruction method combined with multi-planar reconstruction and other original scanned images [9]. Saccular aneurysms showed circular high-intensity shadows in MRA examination, and fusiform aneurysms showed high-intensity curved dilated shadows. Pathological features are based on the angle of rotation plus a variety of complex postprocessing analysis techniques. It takes full advantage of various postprocessing techniques; the original image shows the location, size, shape, tumor neck artery aneurysm, and its relationship to healthy blood vessels. MRA does not affect bones, and it has a good display. It can show internal cavernous carotid artery aneurysms and uses presaturation technology to saturate venous blood flow, avoiding the occurrence of tumor venous contamination. It can not only reflect the anatomical structure of blood vessels but also reflect the changes in hemodynamics, especially for tumor elongated carotid aneurysm or thrombotic aneurysm. CTA performance is significantly better than the former.

The current accepted gold standard for diagnosing cerebrovascular diseases is DSA testing, but it has many shortcomings, such as the following: the inspection takes a long time, it has a lot of $\mathrm{x}$-ray radiation, and it is a slightly traumatic inspection, is prone to complications, and has a certain limitation in display time. In the diagnosis of ischemic cerebral angiography, the examination for atherosclerosis is not as accurate as CTA and MRA. In the diagnosis of aneurysm, it cannot show secondary 
thrombosis in the aneurysm. In addition, DSA cannot reflect the specific relationship between the diseased blood vessel and the surrounding fine tissue structure. However, the two methods of MRA and CTA have their own advantages in detecting cerebrovascular diseases in different types of diseases. Therefore, according to the actual situation of the disease, the specific applicable method is preferred. For the diagnosis of ischemic cerebral angiography, the CTA is adopted to diagnose atherosclerotic plaque. For the diagnosis of aneurysm, since secondary aneurysm thrombosis is basically not present [10-12], DSA cannot detect the specific relationship between the diseased blood vessel and peripheral nerve cells and tissues. Therefore, MRA and CTA have different advantages according to different types of cerebrovascular diseases, so the best choice should be made according to the actual situation.

MRA is a noninvasive angiography technique, which is defined as a two-dimensional or three-dimensional image showing the complete circle of Willis. MRA is highly sensitive to intracranial aneurysms. The previous literature reported that the detection rate of $\mathrm{MRA}$ for $>3 \mathrm{~mm}$ aneurysms was $80 \%-95 \%$, and the specificity was $95 \%-99 \%$. MRA can distinguish aneurysms with a diameter of 3 to $5 \mathrm{~mm}$ or more, which is lower than CTA and DSA. In this study, the smallest diameter of intracranial aneurysms detected by MRA was $5 \mathrm{~mm}$, which was lower than CTA and DSA, and the detection rate of intracranial aneurysms was also lower than CTA and DSA. In the past three years, some researchers used high-resolution MRA images to assess aneurysms, even showing aneurysms with a diameter of $1 \mathrm{~mm}$. Compared with the DSA inspection results, the detection rate was almost the same, both at about $97 \%$. In this study, 150 cases (76 aneurysms) underwent surgical treatment based on MRA examinations only, of which 12 cases were diagnosed as right middle cerebral aneurysm before MRA, and intraoperative evidence was right posterior communicating medial aneurysm [13]. The MRA of 4 cases was normal before operation, and the anterior communicating aneurysm was confirmed during operation. The other 4 patients ( 3 aneurysms) were suspected to be aneurysms by MRA and confirmed by CTA after operation.

CTA is basically an examination that is not harmful to the human body. It is an intravenous injection of a contrast agent in the elbow, and the dose is small, the scanning speed is faster, and the angiography is clearer, so it has a strong reference value for the diagnosis. Previous studies suggested that the sensitivity of CTA to aneurysms was greater than 95.5\%, but CTA also had x-ray radiation, which had high nephrotoxicity and poor reproducibility. However, MRA is noninvasive and nonradioactive and is superior to DSA and CTA in terms of safety and resolution. In particular, the imaging quality of high-intensity MRA is similar to that of DSA, so their combined use will make up for the differences. Many studies revealed that MRA can clearly show the specific changes in the cerebrovascular structure of arteriovenous malformations. However, MRA also has disadvantages. In addition to background suppression, it is also easy to saturate the distal small blood vessels, leading to the loss of peripheral small blood vessels. The incidence of cerebral aneurysms in the population was about $2 \%$ to $7.5 \%$ [14]. Studies found that the detection rate of CTA for cerebral aneurysms was almost the same as 2D DSA, and it was better than 2D DSA in showing tumor shape, tumor neck, and the relationship with tumor vasculature. Studies also showed that the accuracy of CTA to detect cerebral aneurysms was $89.7 \%$, and for cerebral aneurysms with a diameter of $2.5 \mathrm{~mm}>$, it can reach $96.5 \%$, while that of MRA was $88.4 \%$ and $96.8 \%$, respectively [15]. The sensitivity of MRA for the diagnosis of cerebral aneurysms was $92 \%$ and the specificity was $93 \%$. There was no great difference between MRA and 68-slice spiral CT in the characteristics of no ruptured aneurysm. Some scholars pointed out that for cerebral aneurysms without rupture cerebral angiography may not be considered, but MRA can be performed. The diagnostic efficiency of MRA for complete thrombotic aneurysm was also better than cerebral angiography. The results of this study showed that the accuracy of MRA for cerebral aneurysms was $96.75 \%$, and the accuracy of CTA was $91.2 \%$. The accuracy of both was relatively high, and the difference was not statistically considerable.

Cerebrovascular malformation refers to the malformation of the development of the vascular bed in the brain, which is relatively more common in young people, and the most common is arteriovenous malformation [16]. The fatality rate of cerebrovascular malformations was $35.5 \%$. Some scholars revealed that the sensitivity of CTA to cerebrovascular malformations was 91.2 , and the specificity was $76.9 \%$. The sensitivity of MRA was $92.5 \%$ and the specificity was $91.4 \%$. Studies suggested that the effect of MRA on small blood vessels was relatively insufficient. If necessary, more effective blood vessel reconstruction methods should be used to overcome the loss of blood flow signal caused by the spin saturation effect and complex fluid phenomena in the body. The results showed that the diagnostic accuracy rates of MRA and CTA for cerebrovascular malformations were $92.68 \%$ and $94.67 \%$, respectively, and the difference between the two was not remarkable. In short, both MRA and CTA were reliable methods for diagnosing cerebrovascular diseases, and the results were very effective [17-20].

\section{Conclusion}

CTA was the preferred method for the examination of intracranial aneurysms. According to the results of CTA examination, intracranial aneurysms can be directly treated surgically without DSA examination. MRA can be used for the diagnosis of intracranial aneurysms, but the detection rate of aneurysms was lower than CTA and DSA. DSA, CTA, and MRA were three irreplaceable imaging methods. DSA was still the most sensitive and highly accurate inspection method. Although the latter two were not enough to replace DSA, they were the future improvement direction and development trend of neuroimaging and had important reference value in DSA examination. When DSA cannot make a diagnosis or the amount of information it provides cannot meet clinical needs, correct and comprehensive diagnosis can be obtained through joint adoption of detections to learn from each other's advantages. 


\section{Data Availability}

The data used to support the findings of this study are available from the corresponding author upon request.

\section{Conflicts of Interest}

The authors declare no conflicts of interest.

\section{References}

[1] J. Wardlaw, "The detection and management of unruptured intracranial aneurysms," Brain, vol. 1 23, pp. 205-222 2, 2020.

[2] S. Bash and J. Sayre, "Intracranial vascular stenosis and occlusive disease: evaluation with CT angiography, MR angiography and convention angiography," American Journal of Neuroradiology, vol. 21, pp. 1012-1021, 2016.

[3] J. Wardlaw and P. White, "The detection and management of unruptured intracranial aneurysms," Brain, vol. 123, pp. 205-221, 2019.

[4] R. Futatsuya, H. Seto, T. Kamei, A. Nakashima, M. Kurimoto, and S. Endoh, "Clinical utility of 3-dimensional time-offlight magnetic resonance angiography for the evaluation of intracranial aneurysms," Clinical Imaging, vol. 18, pp. 1011069, 2018.

[5] M. Chan, K. Hsiang, S. B. Walkden, W. S. Poon, W. W. Lam, and C. Metreweli, "Detection and assessment of intracranial aneurysms: value of CT angiogram phywitll shaded-surface display," AIR Am genology, vol. 165, pp. 1497-1502, 2017.

[6] K. J. Lee, Y. J. Cho, S. J. Kim et al., "Analysis of the clinicopathologic features of papie microcarcinoma based on 7 $\mathrm{mm}$ tumor features of papillary thyroid microcarcinoma based on 7-mm tumor size," World Journal of Surgery, vol. 35, no. 2, pp. 318-323, 2018.

[7] H. P. Adams, B. H. Bendixen, L. J. Kappelle, J. Biller, B. B. Love, and E. Marsh, "Classification of subtype of acute ischemic stroke, definition for us clinical trial toast. trial of org 10172 acute stroke treatment," Stroke, vol. 24, no. 1, pp. 35-41, 2019.

[8] S. W. Han, S. H. Kim, J. Y. Lee et al., “Anewsubtype class if I cationofis-chemic stroke based on treatment and etiologicm echanism," European Neurology, vol. 5, no. 2, pp. 96-102, 2017.

[9] Y. J. Wang, A. D. Xu, S. Gao, Y. S. Li, and D. Z. Wang, "Chinese ischemic stroke subclassification," Frontiers in Neurology, vol. 15, pp. 2-6, 2018.

[10] J. M. Wardlaw, M. S. Dennis, S. C. Lewis, and L. P. Warlow, "Relationship between pattern of intracranial artery abnormalities on transcranial doppler and oxfordshire community stroke project clinical classification of stroke," Stroke, vol. 31, no. 3, pp. 714-719, 2019.

[11] F. Guerini, M. Acciarresi, G. Agnelli, and M. Paciaroni, "Cryptogenicstroke, time to determine aetiology," Journal of Thrombosis and Haemostasis, vol. 6, no. 4, pp. 549-554, 2018.

[12] T. N. Turan and Z. Rumboldt, "Intracranial atherosclerosis: correlation between in-vivo 3T high resolution MRI and pathology," Atherosclerosis, vol. 237, no. 2, pp. 460-463, 2017.

[13] W. H. Xu and M. L. Li, "Deep tiny flow voids along middle cerebral artery atherosclerotic occlusions a high-resolution MR imaging study," Journal of Neurological Sciences, vol. 339, no. 1-2, pp. 130-133, 2018.

[14] D. Zhao, G. Teng, and X. Chen, "Arterial remodeling in middle cerebral artery atherosclerotic stenosis a high- resolution MRI study," Zhonghua Yixue Zazhi, vol. 94, no. 37, pp. 2893-2896, 2019.

[15] H. Chen, Z. Li, and B. Luo, "Anterior cerebral artery dissection diagnosed using high-resolution MRI," Neurology, vol. 85 , no. 5 , p. $481,2017$.

[16] L. Zhang and C. M. Tian, "Clinical diagnosis of carotid atheroscle rostic plaque hypertensive patients with high resolution magnetic resonance angiography," Journal of Biological Regulators \& Homeostatic Agents, vol. 29, no. 2, pp. 411-415, 2019.

[17] Y. Du and Z. Yang, "Diagnostic value of $m$ spiral CT cardiothoracic combined with angiography in acute chest pain," Journal of Healthcare Engineering, vol. 2021, no. 1, 10 pages, Article ID 5549971, 2021.

[18] F. Mori, F. Ishida, T. Natori et al., "Computational fluid dynamics analysis of lateral striate arteries in acute ischemic stroke using $7 \mathrm{~T}$ high-resolution magnetic resonance angiography," Journal of Stroke and Cerebrovascular Diseases: The Official Journal of National Stroke Association, vol. 28, no. 11, Article ID 104339, 2019.

[19] M. A. Masoomi, I. Al-Shammeri, K. Kalafallah et al., "Wiener filter improves diagnostic accuracy of CAD SPECT imagescomparison to angiography and CT angiography," Medicine, vol. 98, no. 4, Article ID e14207, 2019.

[20] J. Meng, V. M. Mellnick, S. Monteiro, and M. N. Patlas, "Acute aortic syndrome: yield of computed tomography angiography in patients with acute chest pain," Canadian Association of Radiologists Journal, vol. 70, no. 1, pp. 23-28, 2019. 